\title{
EVOLUTIVE INSTANTANEOUS SPECTRUM ASSOCIATED WITH PARTIAL AUTOCORRELATION FUNCTION
}

\author{
By Serge Dégerine and Sophie Lambert-Lacroix \\ Laboratoire LMC-IMAG
}

First Version received September 1998

\begin{abstract}
Several approaches have been developed for the spectral analysis of nonstationary processes in the literature. Otherwise, it has been shown recently that, as in the stationary case, the partial autocorrelation function characterizes, like the autocovariance function, the second-order properties of the process. Our main result is the introduction of a new time-dependent power spectrum clearly related to this function. At each time, this spectrum describes a stationary situation in which the present is correlated with the past in the same way as our nonstationary process at this time. The properties of this spectrum are analysed. In particular, it is defined for all nonstationary processes and is in a one-to-one correspondence with the autocovariance function. Unfortunately, no spectral representation of the process is actually associated with it. This spectrum is also compared with two similar other spectra. Some examples of theoretical spectra and an estimated spectrum are considered for illustration.
\end{abstract}

Keywords. Nonstationary processes; discrete time; second-order properties; partial autocorrelation; time-dependent spectrum.

\section{INTRODUCTION}

The power spectrum is a well-known concept for a stationary process. Indeed, the autocovariance function (ACF) $R(t, s)$, which is a function of $(t-s)$ only, is nonnegative definite (n.n.d). Hence, a spectral measure can be associated with it by the Fourier transform. For nonstationary processes, several attempts to define a spectrum have been developed. Priestley (1965) considers the particular class of oscillatory processes. Such a process may be seen as the result of passing a stationary process through a time-varying filter. So, for a time instant, its evolutionary power spectrum may be interpreted as the classical spectrum of the stationary process which would have been obtained if the filter was fixed. This interpretation, interesting from a physical point of view, leads to slightly different approaches starting directly from the time-domain decomposition of the process. This is the case for the spectrum of Mélard (1978) or the one of Grenier (1984); see also Grenier (1987). Indeed, the former is based on the Wold-Cramér decomposition of the process while the latter is related to an ARMA representation. We also observe another approach in Martin and Flandrin (1985) who establish a Wigner-Ville analysis in the field of the harmonizable processes. 
The main objective of this paper is to describe the covariance structure of a discrete-time random process. The evolutive instantaneous spectrum introduced here is directly related to the partial autocorrelation function (PACF) $\beta(\cdot, \cdot)$. Although the partial correlation notion was introduced many years ago by Yule (1907), the parameterization of a stationary time series given by the PACF is relatively recent (Ramsey, 1974). This result is also observed by Burg (1975), in the signal processing field, where the partial autocorrelation coefficients are called reflection coefficients. In fact, this one-to-one correspondence is a classical result in orthogonal polynomial theory when the spectral measure has an infinite set of growth points (Geronimus, 1960). In particular, that provides the direct correspondence between the PACF and the spectral measure. Recently, the one-to-one correspondence between the ACF and PACF has been extended to the non-stationary situation (Dégerine and Lambert, 1996; Dégerine and LambertLacroix, 2002). As in the stationary case, the advantage of the PACF is that it is subject to very simple constraints in comparison with the ACF which must be n.n.d. The idea developed here, to extend the spectrum concept to the nonstationary case, is based on the following remark. For a fixed $t$ in $\mathbb{Z}$, the function of only one variable,

$$
R_{t}(k)=R(t, t-k) \quad k \geqslant 0
$$

is not necessarily n.n.d. As a consequence, no spectral measure can be associated with $R_{t}(\cdot)$ by the Fourier transform. On the other hand

$$
\beta_{t}(k)=\beta(t, t-k) \quad k \geqslant 0
$$

is always the PACF of a stationary process. So the evolutive instantaneous spectrum is simply defined as the set of measure $\left\{d F_{t}(\lambda), t \in \mathbb{Z}\right\}$, where $d F_{t}(\lambda)$ is the spectral measure associated with $\beta_{t}(\cdot)$. Our approach agrees with the framework of Loynes (1968) on the spectrum concept for nonstationary processes. The evolutive instantaneous spectrum is comparable to those of Mélard and Grenier although these latters are more directly related to the time-domain description of the process rather than to its second-order properties.

The remainder of the paper is organized as follows. Section 2 is devoted to the evolutive instantaneous spectrum. This new spectrum is analysed through the desirable properties of Loynes. In Section 3, the comparison is made with the spectra of Mélard and Grenier. Examples, as the sampled Brownian motion and linear 'chirps', together with the estimated spectrum of an autoregressive process are considered in Section 4 for illustration. Conclusions and further comments on this spectrum are given in Section 5. The paper ends with Section 6 where some proofs can be found.

\section{EVOLUTIVE INSTANTANEOUS SPECTRUM PROPERTIES}

In the first subsection, we review some results on the PACF investigated in Dégerine and Lambert-Lacroix (2002); see also Dégerine and Lambert (1996). 
The evolutive instantaneous spectrum is presented in the second subsection and its analysis through Loynes recommendations is given in the last one.

\subsection{Partial autocorrelation function}

Let $X(\cdot)=\{X(t), t \in \mathbb{Z}\}$ be a scalar complex-valued nonstationary process with zero mean. In this paper, we are concerned only with the second-order properties of the process $X(\cdot)$. Then it is convenient to use a geometrical approach by considering the Hilbert space $\mathcal{M}=\overline{\mathcal{L}}\{X(t), t \in \mathbb{Z}\}$ with the hermitian product $\langle U, V\rangle=E\{U \bar{V}\}$. So the ACF of $X(\cdot)$, denoted by $R(\cdot, \cdot)$, is defined by

$$
R(t, s)=\langle X(t), X(s)\rangle \quad(t, s) \in \mathbb{Z}^{2}
$$

This function satisfies the hermitian symmetry $R(s, t)=\overline{R(t, s)}$. Furthermore, it is n.n.d., that is for all $s \leqslant t$,

$$
R_{s, t}=\{R(s+i, s+j)\}_{i, j=0, \ldots, t-s}
$$

is n.n.d. as the covariance matrix of the random vector $[X(s), \ldots, X(t)]^{\mathrm{T}}$. We consider the decomposition

$$
\mathcal{D}_{R}=\mathcal{I}\left(\mathcal{D}_{R}\right)+\mathcal{B}\left(\mathcal{D}_{R}\right)
$$

of the set $\mathcal{D}_{R}$ of n.n.d. functions, where the interior $\mathcal{I}\left(\mathcal{D}_{R}\right)$ consists of all positive definite (p.d.) functions (all the matrices $R_{s, t}$ are p.d.), while the boundary $\mathcal{B}\left(\mathcal{D}_{R}\right)$ consists of all n.n.d. functions for which some matrix $R_{s, t}$ is singular. In this case, the corresponding process $X(\cdot)$ will be said locally deterministic since for some $s \leqslant t$, the components $X(s), \ldots, X(t)$ are almost surely linearly dependent. In the opposite case, the process $X(\cdot)$ will be said not locally deterministic.

The $(t-s)$ th-order forward partial innovation $\epsilon^{f}(t ; s)$ is the linear prediction error of $X(t)$ by its nearest past of length $t-s$. Putting

$$
\epsilon^{f}(t ; t)=X(t) \quad \text { and } \quad \sigma^{f 2}(t ; s)=\left\|\epsilon^{f}(t ; s)\right\|^{2}
$$

the associated normalized innovation is defined, for $s \leqslant t$, by

$$
\eta^{f}(t ; s)=\frac{\epsilon^{f}(t ; s)}{\sigma^{f}(t ; s)}
$$

with the convention $0^{-1}=0$. Notice that this convention is necessary in the locally deterministic case when $X(t) \in \mathcal{M}(s ; t-1)=\mathcal{L}\{X(s), \ldots, X(t-1)\}$. The backward innovations, obtained by reversing the time index, are denoted with $b$, $\epsilon^{b}(s ; t)$ and $\eta^{b}(s ; t)=\epsilon^{b}(s ; t) / \sigma^{b}(s ; t)$ for $s \leqslant t$. The PACF $\beta(\cdot, \cdot)$ describes, for all $(t, s)$ of $\mathbb{Z}^{2}$, the partial correlation coefficient between $X(t)$ and $X(s)$ in the set $\{X(s), \ldots, X(t)\}$. With the previous notations, we have 


$$
\beta(t, s)= \begin{cases}\left\langle\eta^{f}(t ; s+1), \eta^{b}(s ; t-1)\right\rangle & \text { if } s<t \\ \|X(t)\|^{2} & \text { if } s=t \\ \left\langle\eta^{b}(t ; s-1), \eta^{f}(s ; t+1)\right\rangle & \text { if } s>t\end{cases}
$$

Putting $\beta(t, t)=\operatorname{var}\{X(t)\}$ instead of 1 , the function $\beta(\cdot, \cdot)$, likely to the ACF $R(\cdot, \cdot)$, characterizes the second-order properties of $X(\cdot)$. The advantage of this function is to be easily identifiable. For $t \neq s$, its magnitude is less than 1 , with equality to 1 corresponding to a linear relationships. Indeed, for $s<t,|\beta(t, s)|=1$ if and only if $s$ is the largest integer such that $X(t)$ belongs to the set $\mathcal{M}(s ; t-1)$. By convention, the partial correlation is then put to 0 everywhere it is undefined, i.e. for the points $(t, s-k)$ and $(t+k, s), k \geqslant 1$. In the same way, we have

$$
\beta(t, t-k)=\beta(t+k, t)=0 \text { for } k \geqslant 0
$$

when a variable $X(t)$ is equal to zero almost surely. More precisely, the PACF $\beta(\cdot, \cdot)$ is in the set $\mathcal{D}_{\beta}$ defined by these conditions:

(i) $\beta(s, t)=\overline{\beta(s, t)}$ with $\beta(t, t) \geqslant 0$ and $|\beta(t, s)| \leqslant 1,(t, s) \in \mathbb{Z}^{2}$

(ii) $\beta(t, t)=0 \Rightarrow \beta(t, s)=0, s \in \mathbb{Z}$

(iii) $|\beta(t, s)|=1, s<t \Rightarrow \beta(t, s-k)=\beta(t+k, s)=0, k \geqslant 1$

We also consider the decomposition

$$
\mathcal{D}_{\beta}=\mathcal{I}\left(\mathcal{D}_{\beta}\right)+\mathcal{B}\left(\mathcal{D}_{\beta}\right)
$$

where $\mathcal{B}\left(\mathcal{D}_{\beta}\right)$ consists of all $\beta(\cdot, \cdot) \in \mathcal{D}_{\beta}$ for which (ii) or (iii) is effectively used. Thus $\beta(\cdot, \cdot) \in \mathcal{I}\left(\mathcal{D}_{\beta}\right)$ when, for all $t \in \mathbb{Z}$, it satisfies $\beta(t, t)>0$ and $|\beta(t, s)|<1$ for $s \neq t$.

As it has been shown in Dégerine and Lambert-Lacroix (2002), the set $\mathcal{D}_{\beta}$ is the PACF variation domain. That is, any function of $\mathcal{D}_{\beta}$ is a nonstationary (or stationary) process PACF. This result has been established using an algorithm which allows to construct a process with any prescribed PACF in $\mathcal{D}_{\beta}$. In particular, that implies the surjectivity of the application $R(\cdot, \cdot) \longrightarrow \beta(\cdot, \cdot)$ onto the set $\mathcal{D}_{\beta}$. The injectivity of this application is obtained by extending the LevinsonDurbin algorithm to the general nonstationary situation. Furthermore, $\mathcal{I}\left(\mathcal{D}_{\beta}\right)$ corresponds to the not locally deterministic case while $\mathcal{B}\left(\mathcal{D}_{\beta}\right)$ consists of PACF of the locally deterministic processes.

\subsection{Evolutive instantaneous spectrum definition}

The spectral measure $d F(\lambda)$ of a stationary process $X(\cdot)$ is related to the ACF $R(\cdot)$, where $R(k)=R(t, t-k)$, by the Fourier transform. We have $R(-k)=\overline{R(k)}$ and $R(\cdot)$ is a n.n.d. function. The PACF $\beta(\cdot)$, where $\beta(k)=\beta(t, t-k)$, satisfies $\beta(-k)=\overline{\beta(k)}$ but is subject only to one of these two constraints $(\beta(0)>0)$ : 
(i) $|\beta(k)|<1, \quad k \in \mathbb{Z}^{+}$

(ii) $|\beta(k)|<1,1 \leqslant k<d, \quad|\beta(d)|=1, \quad \beta(k)=0, k>d$

Starting from $\beta(\cdot)$, the Levinson-Durbin algorithm gives $R(\cdot)$, and $d F(\lambda)$ can be obtained by the inverse Fourier transform. When the PACF satisfies the claim (i) above, or equivalently the spectral measure has an infinite set of growth points, the direct correspondence between $\beta(\cdot)$ and $d F(\lambda)$ is provided by the orthogonal polynomials theory (Geronimus, 1960). Let us introduce the space $\mathcal{L}^{2}\{[-\pi, \pi], d F\}$ of complex-valued functions square integrable with respect to the measure $d F$. Then with the hermitian product

$$
\langle\phi, \psi\rangle_{d F}=\int_{-\pi}^{\pi} \phi(\lambda) \overline{\psi(\lambda)} \mathrm{d} F(\lambda)
$$

this set is the Hilbert space $\overline{\mathcal{L}}\left\{\mathrm{e}^{\mathrm{i} n \lambda}, n \in \mathbb{Z}\right\}$. The Gram-Schmidt orthogonalization process of the set $\left\{\mathrm{e}^{\mathrm{i} n \lambda}, n \in \mathbb{N}\right\}$ in the space $\mathcal{L}^{2}\{[-\pi, \pi], d F\}$ defines a system of orthogonal polynomials $\left\{\varphi_{n}^{f}\left(\mathrm{e}^{\mathrm{i} n \lambda}\right), n \in \mathbb{N}\right\}$ called Szegö polynomials. On the other hand, the space $\mathcal{L}^{2}\{[-\pi, \pi], d F\}$ is isometric to $\mathcal{M}$ by the application $\mathcal{I}\left\{\mathrm{e}^{\mathrm{i} n \lambda}\right\}=X(n)$. We have $\epsilon^{f}(n ; 0)=\mathcal{I}\left\{\varphi_{n}^{f}\left(\mathrm{e}^{\mathrm{i} \lambda}\right)\right\}$ and hence

$$
\varphi_{n}^{f}(z)=\sum_{k=0}^{n} a(n, k) z^{n-k} \quad n \in \mathbb{N}
$$

In the backward sense, we obtain $\epsilon^{b}(0 ; n)=\mathcal{I}\left\{\varphi_{n}^{b}\left(\mathrm{e}^{\mathrm{i} \lambda}\right)\right\}$, where $\varphi_{n}^{b}$ is the reciprocal polynomial of $\varphi_{n}^{f}$ :

$$
\varphi_{n}^{b}(z)=\sum_{k=0}^{n} \overline{a(n, k)} z^{k}=z^{n} \overline{\varphi_{n}^{f}}\left(\frac{1}{z}\right)
$$

So the orthogonal system $\left\{\varphi_{n}^{f}, n \in \mathbb{N}\right\}$ is characterized in terms of $\beta(\cdot)$ by the recurrence formulae

$$
\begin{gathered}
\varphi_{0}^{f}(z)=\varphi_{0}^{b}(z)=1 \\
\varphi_{n}^{f}(z)=z \varphi_{n-1}^{f}(z)-\beta(n) \varphi_{n-1}^{b}(z) \\
\varphi_{n}^{b}(z)=z \varphi_{n-1}^{b}(z)-\overline{\beta(n)} z \varphi_{n-1}^{f}(z) \quad n \in \mathbb{N}^{*}
\end{gathered}
$$

In this way, the coefficients $\beta(n), n \geqslant 1$ are related to $d F$, the $[-\pi, \pi]$ measure with respect to $d F$ giving the variance $\beta(0)$. The inverse correspondence is based on the transform which associates to the measure $d F$ the Caratheodory function:

$$
G(z)=\int_{-\pi}^{\pi} \frac{\mathrm{e}^{\mathrm{i} \lambda}+z}{\mathrm{e}^{\mathrm{i} \lambda}-z} \mathrm{~d} F(\lambda) \quad|z|<1
$$

For any $\lambda$ where the function $F$ is continuous, we have

$$
F(\lambda)=\lim _{r \rightarrow 1^{-}} \int_{-\pi}^{\lambda} \mathcal{R}\left\{G\left(r \mathrm{e}^{i \theta}\right)\right\} \mathrm{d} \theta
$$


where $\mathcal{R}\{G(z)\}$ is the real part of $G(z)$. On the other hand, the Caratheodory function is related to $\beta(\cdot)$ by

$$
G(z)=\beta(0) \lim _{n \rightarrow+\infty} \frac{\psi_{n}^{b}(z)}{\varphi_{n}^{b}(z)} \quad|z|<1
$$

where $\left\{\psi_{n}^{f}\left(\mathrm{e}^{\mathrm{i} n \lambda}\right), n \in \mathbb{N}\right\}$ are the polynomials associated to the sequence $\left\{-\beta(n), n \in \mathbb{N}^{*}\right\}$. In the singular case, where the PACF satisfies the claim (ii), the space $\mathcal{L}^{2}\{[-\pi, \pi], d F\}$ is of dimension $d$. The recurrence formulae lead to $(d+1)$ orthogonal polynomials $\left\{\varphi_{n}\left(\mathrm{e}^{\mathrm{i} n \lambda}\right), n=0, \ldots, d\right\}$ with $\left\|\varphi_{d}(\cdot)\right\|=0$. The support of $d F$ is given by the roots of $\varphi_{d}(\cdot)$ and the mass distribution is obtained by algebraic relations.

For a nonstationary process $X(\cdot)$, our goal is to describe, at each time $t$, the correlations between $X(t)$ and its past $\{X(s), s \leqslant t\}$ in some stationary way. Immediately, we want to consider

$$
\rho_{t}(k)=\frac{R(t, t-k)}{\sqrt{R(t, t) R(t-k, t-k)}} \quad k \geqslant 1
$$

but the n.n.d. property of $\rho_{t}(\cdot)$ is generally not satisfied. We overcome this difficulty by using the PACF. Indeed, in the above approach, the correlation between $X(t)$ and $X(t-k)$ can be replaced by the partial correlation between these same variables.

Definition 1. Let $\beta(\cdot, \cdot)$ be the PACF of a process $X(\cdot)$. The evolutive instantaneous spectrum of $X(\cdot)$ is defined as the set of measures $\left\{d F_{t}(\lambda), t \in \mathbb{Z}\right\}$ on $[-\pi, \pi]$ where, for any fixed $t, d F_{t}(\lambda)$ is the spectral measure associated with the $P A C F \beta_{t}(\cdot)$ given by

$$
\beta_{t}(k)=\overline{\beta_{t}(-k)}=\beta(t, t-k) \quad k \geqslant 0
$$

It is easy to see that the functions $\beta_{t}(\cdot)$, so associated to any element of $\mathcal{D}_{\beta}$, are effectively a set of PACF of second-order stationary processes. At each time $t$, $d F_{t}(\lambda)$ is the spectral measure of some stationary process $Y_{t}(\cdot)$, with the same variance as $X(t)$, such that the partial correlation between $Y_{t}(t)$ and $Y_{t}(s)$ is identical to the one between $X(t)$ and $X(s)$ for all $s<t$. Notice that this spectrum is associated to the process in a causal fashion, since $d F_{t}(\lambda)$ depends only on the second-order properties of $\{X(s), s \leqslant t\}$. When $\beta(\cdot, \cdot) \in \mathcal{B}\left(\mathcal{D}_{\beta}\right)$, there exists some relationships between the measures associated with the spectrum. For example, $|\beta(t, s)|=1$ implies $\beta(u, s)=0, u>t$, and hence some constraints on $d F_{u}$. These links between the different measures are not easy to describe because we are unable to characterize the set of measures associated with a PACF which presents one or several zeros. On the other hand, in the not locally deterministic case, $d F_{t}(\lambda)$ does not depend on the structure of the past $\{X(s), s<t\}$, hence the instantaneous character of the spectrum, since $\beta_{t}(\cdot)$ can take any values satisfying $\beta_{t}(0)>0$ and $\left|\beta_{t}(k)\right|<1, k \geqslant 1$. So the spectrum consists of any set of measures $\left\{d F_{t}, t \in \mathbb{Z}\right\}$ having an infinite set of growth points. 
Clearly, different PACF give rise to different evolutive instantaneous spectra. As a consequence, our spectrum allows to determine the PACF of the process and hence its second-order properties.

In the rest of this paper, when one considers several processes, their attributes (innovation, $\mathrm{PACF}, .$. ) will be indexed by the letter representing them.

\subsection{Evolutive instantaneous spectrum properties}

Loynes (1968) proposes two lists of desirable properties for a time-dependent spectrum. The former (from A1 to A8) is rather of physical nature, while the latter (from B1 to B12) is rather of mathematical nature. The evolutive instantaneous spectrum is analysed through these recommendations. Each of its resulting properties will be followed by its Loynes's classification references.

The first property is a simple consequence of the spectrum definition.

Property 1. The evolutive instantaneous spectrum is a real positive function of time and of 'frequency' (Loynes: A1, B1, B8).

Rigorously, our spectrum is defined by a family of measures. When these measures are absolutely continuous with respect to Lebesgue one's, the spectrum consists of the set of corresponding densities $\left\{f_{t}(\lambda)=d F_{t}(\lambda) / d \lambda, t \in \mathbb{Z}\right\}$. In particular, that happens when $\beta_{t}(\cdot)$ belongs to $\ell^{1}$, said $\sum_{k=0}^{+\infty}\left|\beta_{t}(k)\right|<+\infty$.

According to the one-to-one correspondence between $\beta(\cdot, \cdot)$ and $R(\cdot, \cdot)$, the remark following the spectrum definition leads to the next result.

Property 2. The relationship between the evolutive instantaneous spectrum and the $\operatorname{ACF} R(\cdot, \cdot)$ is one-to-one (Loynes: $A 4, B 4)$.

The equality $\operatorname{var}\{X(t)\}=\beta_{t}(0)=\int_{-\pi}^{\pi} d F_{t}(\lambda)$ gives:

Property 3. The evolutive instantaneous spectrum describes the distribution of energy over frequency (Loynes: A2).

For fixed $t$, the measure $d F_{t}$ is the Fourier transform of the ACF of the stationary process which admits $\beta_{t}(\cdot)$ as PACF.

PROPERTy 4. The evolutive instantaneous spectrum is the Fourier transform of some 'apparently meaningful quantity' (Loynes: A8).

The process $X(\cdot)$ is stationary to the second order if and only if $\beta(t, s)$ depends only on $(t-s)$. In this case $\beta_{t}(\cdot)$, which does no longer depend on $t$, is the PACF of $X(\cdot)$. 
Property 5. The evolutive instantaneous spectrum reduces to the ordinary spectrum if $X(\cdot)$ is, in fact, stationary (Loynes: A5, B5).

Loynes wished that if the process is composed of a succession of stationary parts, then the spectrum is also composed of the corresponding succession of stationary spectra (Loynes; A6, B6). Nevertheless, he points out that it is impossible to realize this exactly. On the other hand, one should obtain the spectra in an approximate way. For a stationary process $X(\cdot)$, of PACF $\beta(\cdot)$, we denote by $\mathrm{AR}(\leqslant n)$ the autoregressive model of order less than or equal to $n$ associated with $\{\beta(0), \ldots, \beta(n)\}$ and its spectral measure is denoted by $d F_{n}$. It is well-known that autoregressive spectral estimation provides good accuracy. In particular, $d F_{n}$ coincides with $d F$ when $X(\cdot)$ is linearly singular of order $d$ with $d \leqslant n$ or autoregressive of order $p$ with $p \leqslant n$.

Proposition 1. Let $X(\cdot)$ be a process composed of a succession of stationary parts,

$$
X(t)= \begin{cases}X^{(1)}(t) & \text { if } t \leqslant 0 \\ X^{(2)}(t) & \text { if } t>0\end{cases}
$$

where $X^{(1)}(\cdot)$ and $X^{(2)}(\cdot)$ are two uncorrelated stationary processes. Then we have

$$
d F_{t}^{X}= \begin{cases}d F^{(1)}(t) & \text { if } t \leqslant 0 \\ d F_{t-1}^{(2)}(t) & \text { if } t>0\end{cases}
$$

where $d F^{(1)}$ is the spectral measure of $X^{(1)}(\cdot)$ and $d F_{t}^{(2)}$, is the one of the $\operatorname{AR}(\leqslant t)$ model associated with $X^{(2)}(\cdot)$.

The proof of this proposition appears in Section 6.

The following properties concern elementary transformations on the process $X(\cdot)$.

Property 6. If h is a fixed integer, and $Y(\cdot)$ is defined by shifting $X(\cdot)$ in time by $h, Y(t)=X(t+h)$, then (Loynes: B10),

$$
d F_{t}^{Y}(\lambda)=d F_{t+h}^{X}(\lambda)
$$

This result follows from the equality

$$
\beta^{Y}(t, t-k)=\beta^{X}(t+h, t+h-k)
$$

The proofs of the two following properties appear in Section 6 .

Property 7. If $\lambda_{0}$ is any real number and a new process $Y(\cdot)$ is defined by

$$
Y(t)=X(t) \mathrm{e}^{-\mathrm{i} \lambda_{0} t}
$$

then (Loynes: B9), 


$$
d F_{t}^{Y}(\lambda)=d F_{t}^{X}\left(\lambda+\lambda_{0}\right)
$$

Property 8. (Loynes: B11 $c$ and $d)$

(i) If $Y(t)=\overline{X(t)}, t \in \mathbb{Z}$, then $d F_{t}^{Y}(\lambda)=d F_{t}^{X}(-\lambda), t \in \mathbb{Z}$.

(ii) If $X(t) \in \mathbb{R}, t \in \mathbb{Z}$, then $d F_{t}^{X}(\lambda)=d F_{t}^{X}(-\lambda), t \in \mathbb{Z}$.

The claims (a) and (b) of property B11 considered by Loynes concern the time reversal. Here, they are generally false. According to the point (i) of Property 8, they can be written in the alternative forms

(a) $\forall t \in \mathbb{Z}, Y(t)=\overline{X(-t)} \Rightarrow \forall t \in \mathbb{Z}, d F_{t}^{Y}(\lambda)=d F_{-t}^{X}(\lambda)$

(b) $\forall t \in \mathbb{Z}, Y(t)=X(-t) \Rightarrow \forall t \in \mathbb{Z}, d F_{t}^{Y}(\lambda)=d F_{-t}^{X}(-\lambda)$

The definition of $Y(\cdot)$ in (a) corresponds in terms of PACF to $\beta_{t}^{Y}(k)=\beta_{-t+k}^{X}(k)$ for all $t \in \mathbb{Z}$ and $k \geqslant 0$. Moreover, the condition $d F_{t}^{Y}(\lambda)=d F_{-t}^{X}(\lambda)$ is equivalent to $\beta_{t}^{Y}(k)=\beta_{-t}^{X}(k), k \geqslant 0$. So these properties will be true if and only if $\beta_{t}^{X}(k)=\beta_{t+k}^{X}(k)$ for all $t \in \mathbb{Z}$ and all $k \geqslant 0$. In particular, this result arises in the stationary case.

Finally, we comment on two important properties which cannot agree exactly with our spectrum.

(i) The spectrum is a linear transform of the ACF (Loynes: B2).

(ii) The spectrum transforms reasonably when $X(\cdot)$ is transformed linearly (Loynes: $A 3, B 3)$.

These properties concern operations on $X(\cdot)$ which are simply expressed on $R(\cdot, \cdot)$. For example, it relates to the sum of two uncorrelated random processes in the case (i), or a process passing through a time varying filter in the case (ii). Using simple examples, we observe that the effects on the evolutive instantaneous spectrum are immediately very complicated. That comes from the fact that the correspondence between $R(\cdot, \cdot)$ and $\beta(\cdot, \cdot)$ is highly nonlinear, and it is also the case between $\beta(\cdot, \cdot)$ and $d F_{t}(\lambda)$. Nevertheless, recall that these correspondences are oneto-one, so the transformed spectrum is always computable.

To end this study, we have to mention that the spectrum depends continuously on the covariance (Loynes: B12) and consider the problem of its estimation (Loynes: A7, B7). For the first claim, it is not clear, as noticed by Loynes, how continuity should be understood. Nevertheless, the one-to-one correspondence between $R(\cdot, \cdot)$ and $\beta(\cdot, \cdot)$, given by the extended Levinson-Durbin algorithm, uses only very simple algebraic relations. It is also the case between $\beta_{t}(\cdot)$ and $R_{t}(\cdot)$ (not $R(t, t-\cdot)$ ) for each time $t$. Finally $d F_{t}(\cdot)$ is related to $R_{t}(\cdot)$ by the usual Fourier transform. So, all tools are clearly available to study any kind of continuity.

Concerning estimation, the problem is meaningful uniquely for some particular classes of processes insofar as we observe only one record, even of infinite length. 
Without spectral representation associated with our spectrum, and taking into account its definition in terms of second-order characteristics in the time domain, a natural estimation is given by the autoregressive spectral estimator, computed on a sliding window, or by any spectral estimator used in the stationary case. An illustration for an $\mathrm{AR}(1)$ process with time-dependent coefficient is given in Section 4.

\section{TWO SIMILAR SPECTRA}

In this section, we consider the spectrum of Mélard (1978) and the one of Grenier (1984) which are the closer ones to the evolutive instantaneous spectrum. After presenting them, the comparison with our spectrum is made through the Loynes's properties.

\subsection{The evolutive spectrum}

The evolutive spectrum proposed by Mélard (1978) is based on the WoldCramér decomposition (Cramér, 1961). Notice that this spectrum also has been introduced by Tjøstheim (1976), but in a different way. Let us consider a purely nondeterministic process $X(\cdot)$. Then its Wold-Cramér decomposition is given by

$$
X(t)=\sum_{j=0}^{+\infty} h(t, t-j) \eta(t-j)
$$

where $\eta(\cdot)$ is the normalized innovation process. Then the process $X(\cdot)$ admits a representation of the form

$$
X(t)=\int_{-\pi}^{\pi} \psi_{t}(\lambda) \mathrm{d} \xi(\lambda)
$$

where

$$
\psi_{t}(\lambda)=(2 \pi)^{-1 / 2} \sum_{j=0}^{+\infty} h(t, t-j) \mathrm{e}^{\mathrm{i} \lambda(t-j)}
$$

and $\xi(\cdot)$ has uncorrelated increments with $E\left\{|d \xi(\lambda)|^{2}\right\}=d \lambda$. This representation is the analogous of the spectral representation of the stationary case and the ACF admits the one given by

$$
R(t, s)=\int_{-\pi}^{\pi} \psi_{t}(\lambda) \overline{\psi_{s}(\lambda)} \mathrm{d} \lambda
$$

So, the evolutive spectrum $f_{t}(\lambda)$ is defined as 


$$
f_{t}(\lambda)=\left|\psi_{t}(\lambda)\right|^{2}=\frac{1}{2 \pi}\left|\sum_{j=0}^{+\infty} h(t, t-j) \mathrm{e}^{\mathrm{i} \lambda j}\right|^{2}
$$

Notice that the evolutive instantaneous spectrum is directly defined from the PACF. Unfortunately, no spectral representation of $X(\cdot)$ is actually associated with it. In Mélard (1978), the author analyses his spectrum through the desirable properties of Loynes. It presents, as a whole, the same properties as our spectrum. However, we record basic differences. Concerning the linear transformation (Loynes: A3), Mélard obtains an approximate result, but in a very particular situation. We already pointed out the difficulty of this problem in Section 2. This difficulty is also apparently related to the problem of the process spectral representation. Relating to the advantages presented by our spectrum, one can notice that it is defined for any nonstationary process whereas the Mélard's one only exists for the class of purely nondeterministic processes.

A fundamental difference between these two definitions is that the evolutive spectrum of Mélard is no longer in one-to-one correspondence with the ACF. Hence, contrarily to the stationary case, this spectrum no longer constitutes another parameterization of the second-order properties of the process. In fact, the same evolutive spectrum can be associated with two processes having different second-order properties. This point is illustrated in Mélard (1978) by the following example. Given a white noise $\epsilon(\cdot)$ with variance 1 , let us generate a process $X(\cdot)$,

$$
X(t)= \begin{cases}\epsilon(t)-0.5 \epsilon(t-1) & \text { if } t \neq 1 \\ 0.5 \epsilon(1)-\epsilon(0) & \text { if } t=1\end{cases}
$$

Then $\epsilon(\cdot)$ is the normalized innovation process. Therefore, the evolutive spectrum of $X(\cdot)$ is given by the spectral density of the stationary process $Y(t)=\epsilon(t)-0.5 \epsilon(t-1)$, while their covariances are different. An unfortunate consequence, which has not been pointed out by the author, is that an evolutive spectrum unchanging with $t$ time does not necessarily imply the process under study to be stationary. Recall that the correspondence between the class of the evolutive instantaneous spectra and the class of ACF is one-to-one. In particular, an evolutive instantaneous spectrum unchanging with $t$ time corresponds to the stationarity of the process under study since, in this case, the function $\beta_{t}(\cdot)$ does not depend on time. Notice that, in the previous example, for $t<1$, our spectrum is given by the one of the stationary process $Y(\cdot)$. Otherwise, it depends on time and coincides again with the one of $Y(\cdot)$ only as $t$ goes to infinity.

Finally, the last remark about this spectrum concerns the nonstationary processes of the form

$$
X(t)= \begin{cases}X^{(1)}(t) & \text { if } t \leqslant 0 \\ X^{(2)}(t) & \text { if } t>0\end{cases}
$$

where both $X^{(1)}(\cdot)$ and $X^{(2)}(\cdot)$ are stationary but with different ACF. As our spectrum, $f_{t}(\cdot)$ corresponds to the spectral density of the first process for $t \leqslant 0$. 
When the both processes have the same normalized innovation $\eta(\cdot)$, we obtain the spectral density of the second process for $t>0$. This is no longer true for our spectrum. Now, let us assume that the both processes are uncorrelated. A result similar to Proposition 1 does not exist for the evolutive spectrum of Mélard. Furthermore, if $X^{(2)}(\cdot)$ is autoregressive of order $p$, then $f_{t}(\cdot)$ corresponds to its spectral measure only for an infinite time. Recall that this time is finite and equal to $p+1$ for our spectrum.

\subsection{The rational spectrum}

Following the evolutive spectrum of Mélard, Grenier (1984) - see also Grenier (1987) - wished a spectrum more adapted to the previous case of the succession of stationary parts. For this, he proposes a more instantaneous spectrum based on the existence of the ARMA representation. So, let $X(\cdot)$ be a purely nondeterministic process which admits the $\operatorname{ARMA}(p, q)$ representation

$$
\sum_{j=0}^{p} a_{j}(t-j) X(t-j)=\sum_{j=0}^{q} b_{j}(t-j) \eta(t-j)
$$

where $\eta(\cdot)$ is the normalized innovation process. So, for each fixed $t$, the rational spectrum $\rho(t, \lambda)$ is defined as the spectral density of the 'stationary' process which admits the ARMA representation (2), namely

$$
\rho(t, \lambda)=\frac{1}{2 \pi} \frac{\left|\sum_{j=0}^{q} b_{j}(t-j) \mathrm{e}^{-\mathrm{i} j \lambda}\right|^{2}}{\left|\sum_{j=0}^{p} a_{j}(t-j) \mathrm{e}^{-\mathrm{i} j \lambda}\right|^{2}}
$$

Notice that the word stationary above is written in inverted commas because, for a fixed $t$, the parameters $a_{j}(t-j), j=1, \ldots, p$ and $b_{j}(t-j), j=0, \ldots, q$, are not necessarily those of a stationary ARMA model (as will be seen in the next section).

Using very simple examples, it is easy to see that the rational spectrum is different from ours. Now, we propose to compare them through the desirable properties of Loynes.

Recall that Grenier has introduced his spectrum so as to improve the one of Mélard relating to the succession of stationary parts. The rational spectrum seems to be better adapted to this request among the three spectra. Indeed, when the stationary parts have the same innovation process, this spectrum is exactly composed of the succession of the correspondent spectral densities. On the other hand, if the stationary processes are uncorrelated, the same result holds except over adaptation zones of duration equal to $\max (p, q+1)$. Notice that this last property remains valid for our spectrum in the autoregressive case, and otherwise, it consists of the autoregressive approximations of the spectral measures (see Proposition 1).

Unfortunately, this improvement for the rational spectrum is achieved with the loss of Property A2 of Loynes, requiring the spectrum to describe the distribution 
of energy over frequency. As a matter of fact, let us consider the example given in Grenier (1984); see also Grenier (1987). Let $X(\cdot)$ be a process which admits a representation of the form (2) with $q=0$ :

$$
\begin{gathered}
X(t)+a_{1}(t-1) X(t-1)=\eta(t) \\
a_{1}(t)= \begin{cases}-\alpha & \text { if } t<0 \text { or } t>T \\
-\frac{1}{\alpha} & \text { if } t \in[0, T]\end{cases}
\end{gathered}
$$

where $\alpha$ is a real constant satisfying $|\alpha|<1$. Then the variance of $X(t)$ is

$$
\operatorname{var}\{X(t)\}= \begin{cases}\frac{1}{1-\alpha^{2}} & t \leqslant 0 \\ \frac{\alpha^{2}\left(\alpha^{-2(t+1)}+\alpha^{-2 t}-1\right)}{1-\alpha^{2}} & 1 \leqslant t \leqslant T+1 \\ \frac{1+\alpha^{2(t-T-1)}\left(\alpha^{-2(T+1)}+\alpha^{-2 T}-\alpha^{2}-1\right)}{1-\alpha^{2}} & t \geqslant T+2\end{cases}
$$

It follows that the variance of the process depends on time over the two intervals $[1, T+1]$ and $\left[T+2,+\infty\left[\right.\right.$. As a consequence, it cannot be equal to $\int_{-\pi}^{\pi} \rho(t, \lambda) \mathrm{d} \lambda$ since this last quantity is unchanging over these intervals.

Otherwise, the answers related to this spectrum to the other requirements of Loynes are essentially similar to the one of Mélard. The remarks about it are like those about the evolutive spectrum pointed out in subsection 3.1. In particular, the correspondence between the class of rational spectra and that of ACF is still not one-to-one.

\subsection{Remark}

Priestley and Tong (1969) have extended the original definition of oscillatory spectrum to the multivariate case. This has been done also for the evolutive spectrum by Mélard and Herteleer-De Schutter (1989) and, from multivariate ARMA theory, the case of the rational spectrum is straightforward. The PACF of a stationary multivariate process has been introduced by Dégerine (1990). The extension to the nonstationary case can be done without difficulties for regular processes and the definition of the evolutive instantaneous spectrum follows immediately. Unfortunately, this spectrum depends on the chosen PACF which is not uniquely defined in the multivariate case. So, a careful study of this problem is beyond the scope of this paper.

\section{SOME ILLUSTRATIONS}

Here some examples illustrate the behaviour of the evolutive instantaneous spectrum. It will be compared with the spectrum of Mélard and the one of 
Grenier when possible. Indeed, recall that both these spectra are not always defined.

\subsection{The uniformly modulated process}

One interesting example of nonstationarity is given by the uniformly modulated processes. These processes have been studied by Herbst (1963). They are defined by

$$
Y(t)=c(t) X(t) \quad t \in \mathbb{Z}
$$

where $X(\cdot)$ is a stationary process with zero mean and spectrum $d F^{X}(\lambda)$ and $c(\cdot)$ is a real positive function. For this particular processes class, the evolutive instantaneous spectrum behaves well as the one of Priestley (1965), namely

$$
d F_{t}^{Y}(\lambda)=c^{2}(t) d F^{X}(\lambda)
$$

In effect, the PACF of $Y(\cdot)$ is equal to that of $X(\cdot)$ at any point $(t, s)$ where $s \neq t$, and the variances satisfy $\beta_{t}^{Y}(0)=c^{2}(t) \beta^{X}(0)$.

Considering the purely not deterministic processes, the same result is obtained for the spectrum of Mélard, since the coefficients of the Wold-Cramer decomposition of $Y(\cdot)$ are given by $h^{Y}(t, t-j)=c(t) h^{X}(j)$. On the other hand, this is no longer true for the rational spectrum. Suppose that the process $X(\cdot)$ admits an $\operatorname{ARMA}(p, q)$ representation with parameters $a_{j}, j=0, \ldots, p$ and $b_{j}, j=0, \ldots, q$, and that the function $c(\cdot)$ does not vanish. Then the rational spectrum is given by

$$
\rho(t, \lambda)=\frac{\frac{\sigma_{\epsilon}^{2}}{2 \pi}\left|\sum_{j=0}^{q} b_{j} \mathrm{e}^{-\mathrm{i} \lambda j}\right|^{2}}{\left|\sum_{j=0}^{p} \frac{1}{c(t-j)} a_{j} \mathrm{e}^{-\mathrm{i} \lambda j}\right|^{2}}=c^{2}(t) \frac{\frac{\sigma_{\epsilon}^{2}}{2 \pi}\left|\sum_{j=0}^{q} b_{j} \mathrm{e}^{-\mathrm{i} \lambda j}\right|^{2}}{\left|\sum_{j=0}^{p} \frac{c(t)}{c(t-j)} a_{j} \mathrm{e}^{-\mathrm{i} \lambda j}\right|^{2}} \quad a_{0}=b_{0}=1
$$

The second expression of $\rho(t, \lambda)$ above shows clearly that the property is not satisfied.

\subsection{The sampled Brownian motion}

Let $Y(\cdot)=\left\{Y(t), t \in \mathbb{R}^{+}\right\}$be a standard Brownian motion. Such a process is zero mean and its second-order properties are characterized by

$$
\mathrm{E}\{Y(t) Y(s)\}=\inf (t, s)
$$

In particular, it vanishes almost surely at 0 time. We consider the sampled process $X(\cdot)$ obtained in the following way 


$$
X(t)= \begin{cases}0 \text { p.s. } & \text { if } t \leqslant 0 \\ Y(t h) & \text { otherwise }\end{cases}
$$

which $h$ is a real strictly positive constant. The ACF of $X(\cdot)$ satisfies $R(t, s)=$ $\sup (0, h \inf (t, s))$. The random walk defined by $\sum_{k=0}^{t} \epsilon(k)$ if $t>0$, otherwise equal to zero, where $\epsilon(\cdot)$ is a white noise with variance $h$, has the same ACF as $X(\cdot)$. So, the latter admits the autoregressive representation

$$
X(t)-X(t-1)=\epsilon(t)
$$

where $\epsilon(\cdot)$ is the innovation process of variance $\sigma_{\epsilon}^{2}(t)=h$ for $t>0$, and 0 otherwise. Then the PACF $\beta_{t}(\cdot)$ of the process is equal to zero beyond 1. Using the generalized Levinson-Durbin Algorithm (Dégerine and Lambert-Lacroix, 1996), the first coefficients are given by

$$
\begin{gathered}
\beta_{t}(0)=\sup (0, h t) \\
\beta_{t}(1)=\frac{R(t, t-1)}{[R(t-1, t-1) R(t, t)]^{\frac{1}{2}}}=\sqrt{\frac{t-1}{t}}
\end{gathered}
$$

if $t>0$, and 0 otherwise. So, the evolutive instantaneous spectrum of $X(\cdot)$ is equal to zero for $t \leqslant 0$. For $t>0$, it is equal to the spectral measure of the stationary AR(1) model with parameters

$$
\begin{gathered}
\alpha_{t}(1)=-\beta_{t}(1)=-\sqrt{\frac{t-1}{t}} \\
\sigma_{\epsilon, t}^{2}=\left[1-\left|\beta_{t}(1)\right|^{2}\right] \beta_{t}(0)=\sigma_{\epsilon}^{2}(t)=h
\end{gathered}
$$

namely

$$
d F_{t}(\lambda)=\frac{h}{2 \pi}\left|1-\sqrt{\frac{t-1}{t}} \mathrm{e}^{-\mathrm{i} \lambda}\right|^{-2} d \lambda
$$

Notice that, as $t$ goes to infinity, the pole at $\sqrt{(t-1) / t}$ converges to 1 . Then the corresponding normalized spectral measure, which no longer depends on $h$, converges to the Dirac measure at $\lambda=0$ with a spectral density going uniformly to zero over any compact which does not contain 0 .

The process $X(\cdot)$ is purely nondeterministic and its Wold-Cramér decomposition is given by

$$
X(t)=\sum_{j=0}^{t-1} h^{\frac{1}{2}} \eta(t-j)
$$


if $t>0$, and 0 otherwise. The evolutive spectrum is equal to 0 for $t \leqslant 0$ and for $t>0$,

$$
f_{t}(\lambda)=\frac{h}{2 \pi}\left|\sum_{j=0}^{t-1} \mathrm{e}^{-\mathrm{i} \lambda j}\right|^{2}= \begin{cases}\frac{h}{2 \pi} \frac{\left|1-\mathrm{e}^{-\mathrm{i} \lambda t}\right|^{2}}{\left|1-\mathrm{e}^{-\mathrm{i} \lambda}\right|^{2}} & \text { if } \lambda \neq 0 \\ \frac{h t^{2}}{2 \pi} & \text { if } \lambda=0\end{cases}
$$

This is the spectral density of a moving average of order $t-1$ for which all roots lie uniformly on the unit circle: $z=\mathrm{e}^{2 \mathrm{i} k \pi / t}, k=1, \ldots, t-1$ (Figure 1). When $t$ goes to infinity, the normalized spectrum converges to the Dirac measure at $\lambda=0$, like our spectrum, but with a very irregular spectral density. For this particular example, the ACF $R_{t}(\cdot)$ associated to $f_{t}(\lambda)$ is given by the covariances between $X(t)$ and its past: $R_{t}(k)=R(t, t-k), k \geqslant 0$ and $R_{t}(k)=(t-k) h$ if $0 \leqslant k<t, 0$ if $k \geqslant t$. This is no longer true in terms of autocorrelation. That shows that the spectrum associated with $R_{t}(\cdot)$, when defined, can be very different from the evolutive instantaneous spectrum related to $\beta_{t}(\cdot)$.

For $t$ strictly positive, the process $X(\cdot)$ admits the representation $\operatorname{ARMA}(0,1)$ of the form (2) with $a_{1}(t)=-1$ and $b_{0}(t)=h^{1 / 2}$. So the rational spectrum, equal to zero for $t \leqslant 0$, is given by

$$
\rho(t, \lambda)=\frac{h}{2 \pi}\left|1-\mathrm{e}^{-\mathrm{i} \lambda}\right|^{-2} \quad t>0
$$

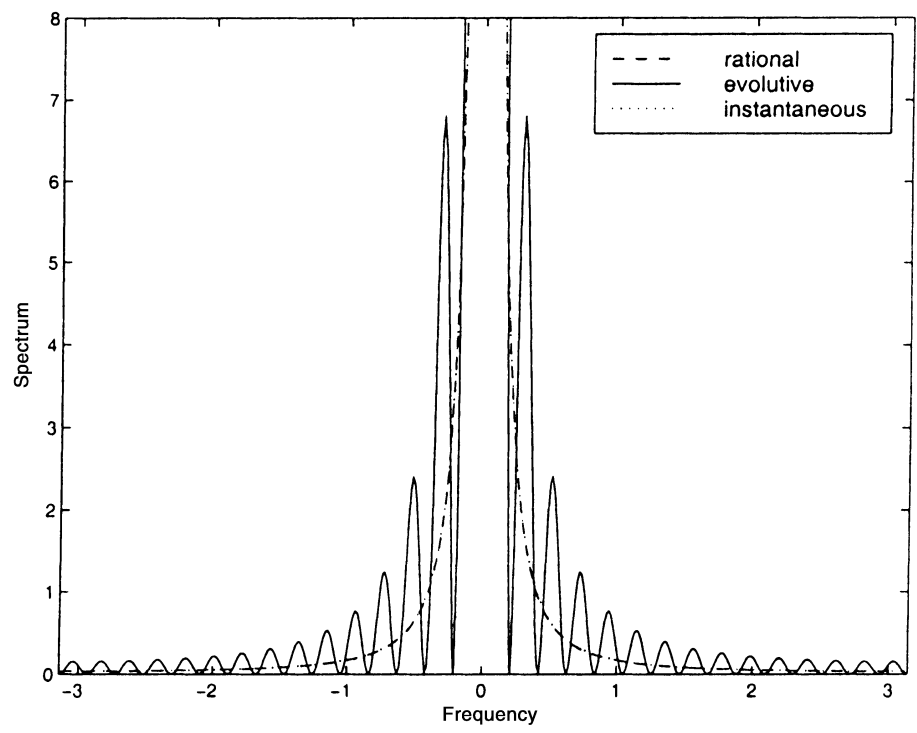

Figure 1. Plot of the rational spectrum, the evolutive spectrum and the evolutive instantaneous spectrum with $t=30, \lambda \in[-\pi, \pi], h=1$. Here $\rho(t, \lambda)$ (dashed line) and $d F_{t}(\lambda) / d \lambda$ (dotted line) seem to be identical (dashdot line). 
This example illustrates two failings of the rational spectrum: it does not depend on time while the process is not stationary and, furthermore, it does not provide the variance of $X(t)$ since this 'spectral density' is not integrable. As $t$ goes to infinity, it corresponds to the limit of the spectral density of the evolutive instantaneous spectrum (without normalization). The behaviour of these three spectra is illustrated by the Figure 2 .

\subsection{Linear chirps}

We consider the process

$$
Z(t)=\mathrm{e}^{\mathrm{i}\left[v_{0} t^{2}+\lambda_{0} t+\Phi\right]} \quad t \in \mathbb{Z}
$$

where $v_{0}$ and $\lambda_{0}$ are real constants and $\Phi$ is uniformly distributed on $\left.]-\pi, \pi\right]$. In the complex plane, $Z(t)$ describes the evolution of the position of a mobile on the circle. The hazard $\Phi$ indicates its position at 0 time and the rotational speed is equal to $2 v_{0} t+\lambda_{0}$. That linear variation should be expresses by the spectrum if it is a good representation of the instantaneous frequency. Notice that the process $Z(\cdot)$ is deterministic with

$$
Z(t)=\mathrm{e}^{\mathrm{i}\left[v_{0}(2 t-1)+\lambda_{0}\right]} Z(t-1)
$$

So $f_{t}(\lambda)$ and $\rho(t, \lambda)$ are not defined for this example.

According to Property 7 , the effect of the constant $\lambda_{0}$ implies, on the spectrum, a shift in frequency by $\lambda_{0} \bmod 2 \pi$ belonging to $\left.]-\pi, \pi\right]$. On the other hand, we can take $v_{0}$ in $\left.]-\pi / 2, \pi / 2\right]$. For $\lambda_{0}=0$, the $\mathrm{ACF}$ of $Z(\cdot)$ satisfies $R(t, s)=\mathrm{e}^{\mathrm{i} v_{0}\left(t^{2}-s^{2}\right)}$, hence it follows that $\beta_{t}(0)=1$ and $\beta_{t}(1)=\mathrm{e}^{\mathrm{i} v_{0}(2 t-1)}$. Since the magnitude of the latter coefficient is equal to 1 , the function $\beta_{t}(\cdot)$ vanishes beyond 1. So, the spectral measure $d F_{t}$ reduces to the Dirac measure at the frequency of $]-\pi, \pi]$ equal to $v_{0}(2 t-1) \bmod 2 \pi$. Then, the support of the evolutive instantaneous spectrum of $Z(\cdot)$ is on lines with slope $2 v_{0} \bmod 2 \pi$ in the set $\mathbb{Z} \times]-\pi, \pi]$. Figure 3 represents this spectrum in the time-frequency plane.

\subsection{An estimated spectrum}

We consider the process

$$
X(t)= \begin{cases}0 \text { p.s. } & \text { if } t<0 \\ a_{t} X(t-1)+b_{t} \eta(t) & \text { otherwise }\end{cases}
$$

where

$$
a_{t}=1-\exp \left(\frac{-t}{100}\right)
$$



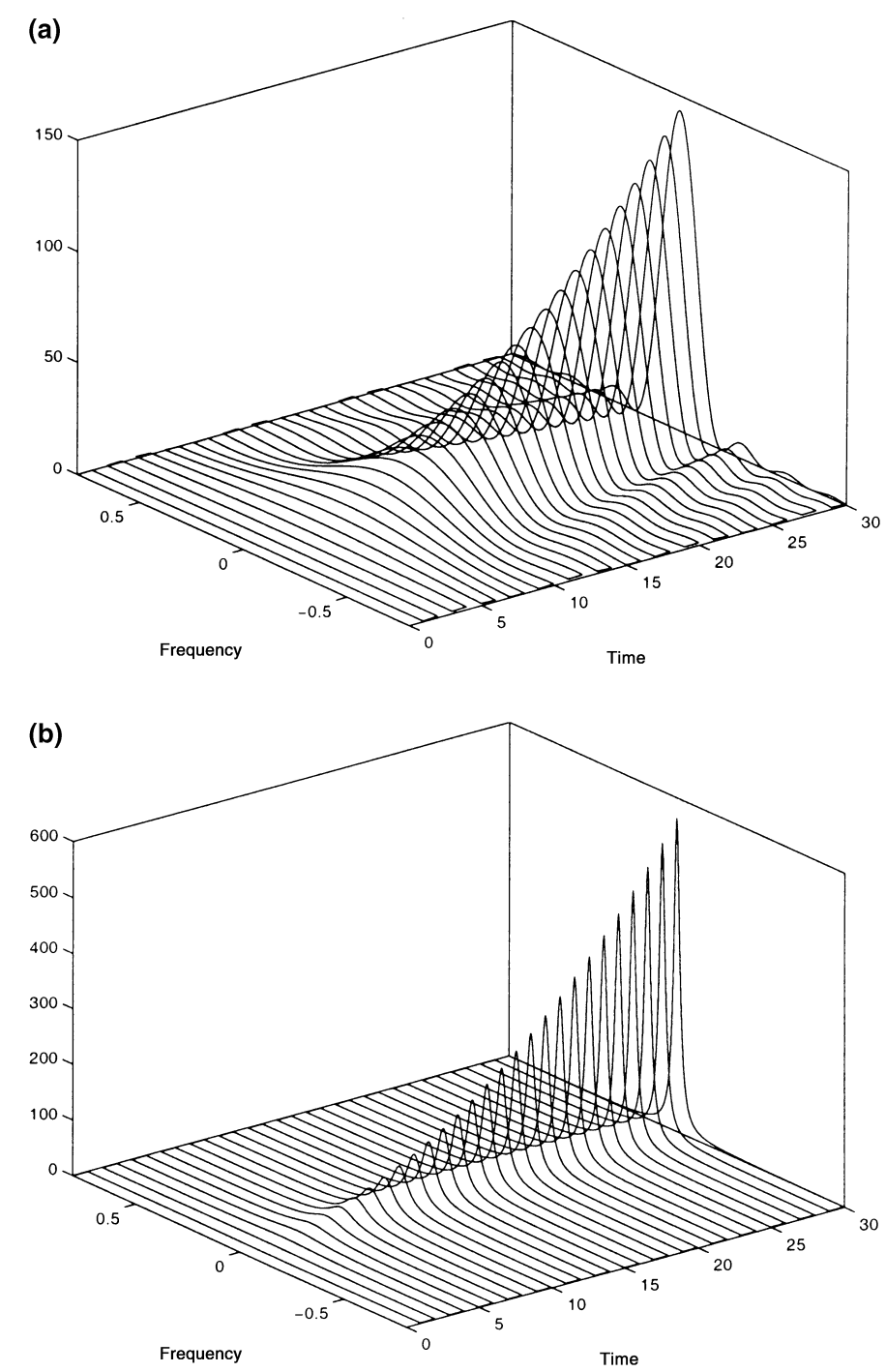

FiguRE 2. Three-dimensional plot of (a) the evolutive spectrum and (b) the evolutive instantaneous spectrum, with $t \in[0, \ldots, 30], \lambda \in[-0.8,0.8], h=1$.

$$
b_{t}=\sqrt{2 \exp \left(\frac{-t}{100}\right)-\exp \left(\frac{-2 t}{100}\right)}
$$

and $\eta(t), t=0,1, \ldots$, are i.i.d. Gaussian random variables with $E \eta(t)=0$ and $E \eta(t)^{2}=1$. 


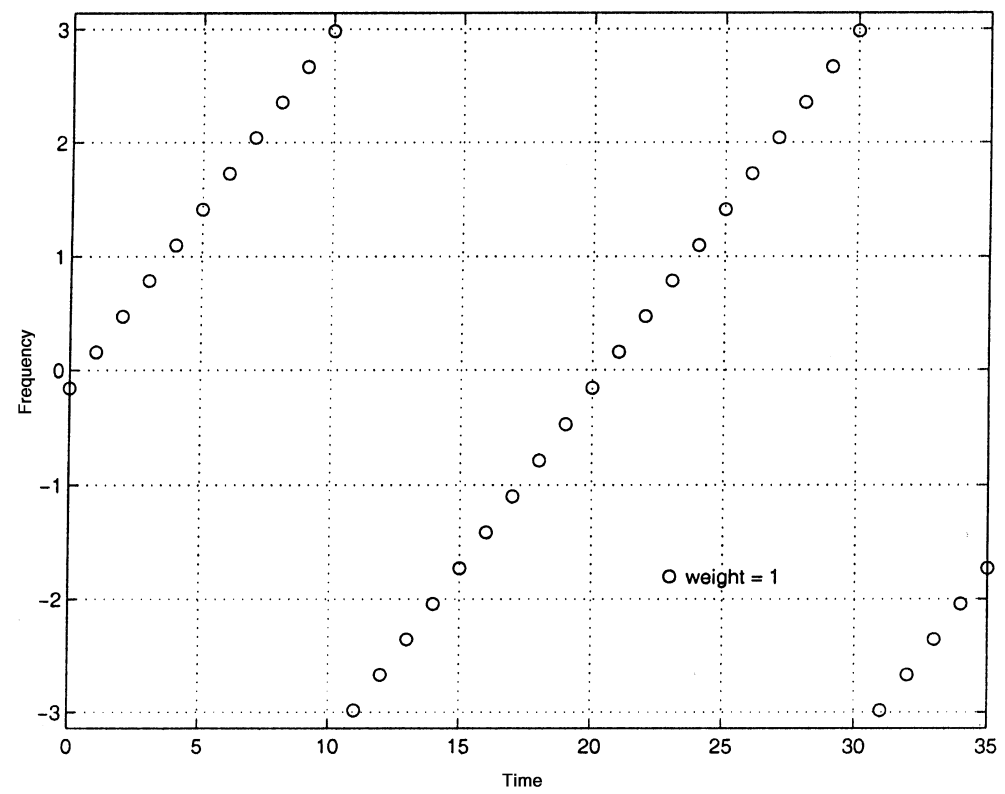

Figure 3. Time-frequency plot of the evolutive instantaneous spectrum with $v_{0}=\pi / 20, \lambda_{0}=0$.

The first coefficients of the corresponding PACF are given by $\beta_{t}(0)=1$, $\beta_{t}(1)=a_{t}$ for $t=0,1, \ldots$ So, the evolutive instantaneous spectrum of $X(\cdot)$ is composed of the corresponding succession of AR(1) model spectra (Figure 4).

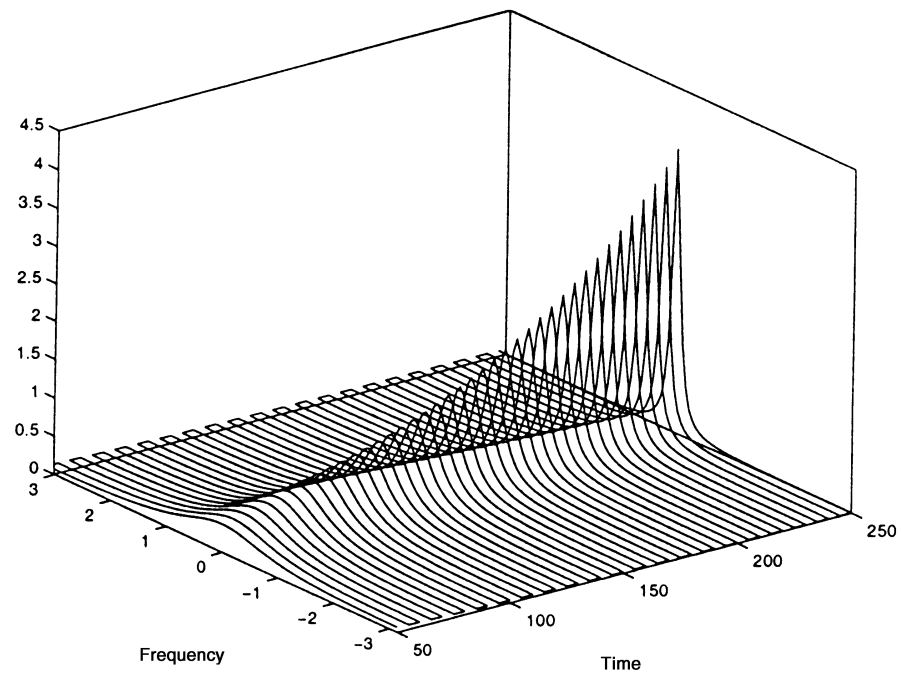

FIgURE 4. Three-dimensional plot of the evolutive instantaneous spectrum with $t \in[50, \ldots, 250]$, $\lambda \in[-\pi, \pi]$. 


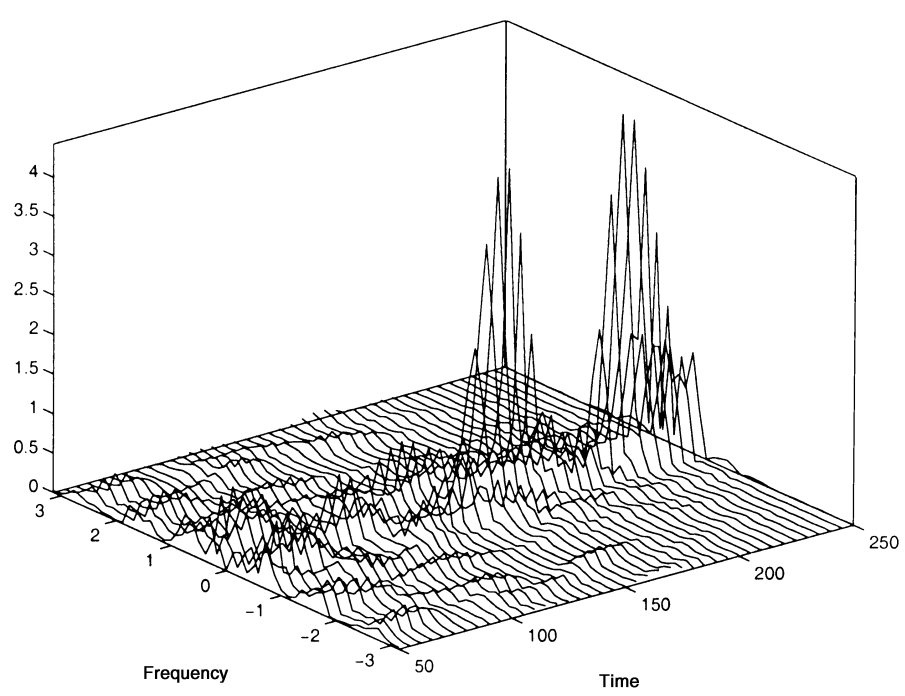

FIGURE 5. Three-dimensional plot of the evolutive instantaneous spectrum estimate obtained from one realization, $t \in[50, \ldots, 250], \lambda \in[-\pi, \pi]$.

Notice that the rational spectrum and our spectrum are the same (since $1-a_{t}^{2}=b_{t}^{2}$ ). Concerning the evolutive spectrum, it is given by

$$
f_{t}(\lambda)=\frac{1}{2 \pi}\left|\sum_{j=0}^{t} h(t, t-j) \mathrm{e}^{-\mathrm{i} \lambda j}\right|^{2}
$$

with

$$
h(t, t-j)=b_{t-j} \prod_{k=0}^{j-1} a_{t-k} \quad \prod_{k=0}^{-1}=1
$$

However, no differences appear when drawing these three spectra. Due to the nature of these spectra, a natural estimation can be obtained by some spectral estimator of the stationary case, computed on a sliding window. Artificial realizations of the process were constructed and estimates of $d F_{t}(\lambda) / d \lambda$ were obtained for $t=50,55, \ldots, 250$, with a window bandwidth $h=50$. For such $t$, the spectrum is estimated by the power spectral density using Welch's method averaged, modifies periodogram method (standard psd function of Matlab signal processing toolbox). Figure 5 shows the spectrum estimated from one realization. We have noticed a large variance but the average behaviour of this estimator seems to be good (Figure 6). 


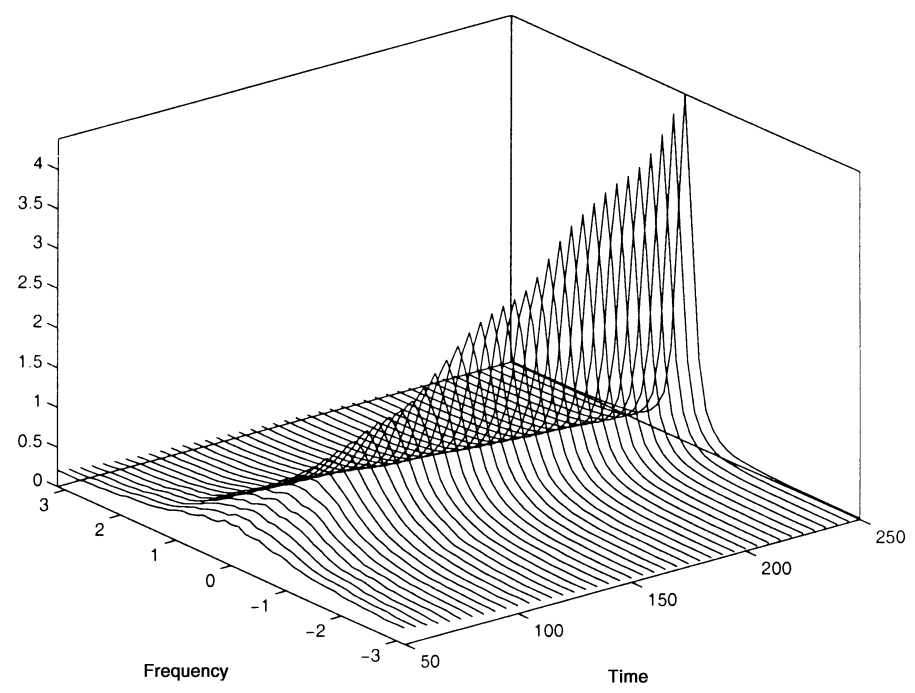

Figure 6. Three-dimensional plot of the mean of the estimated evolutive instantaneous spectrum obtained from 1000 realizations, $t \in[50, \ldots, 250], \lambda \in[-\pi, \pi]$.

\section{CONCLUDING REMARKS}

In this paper, a new time-varying power spectrum, called evolutive instantaneous spectrum, has been naturally associated with the parameterization given by the PACF. This spectrum has been analysed through the desirable properties of Loynes, compared with two similar spectra and illustrated by some examples. We have observed that it is defined for any nonstationary process and is in a one-toone correspondence with the ACF. Unfortunately, we cannot hope, when the process is transformed linearly, that the resulting spectrum is a simple transform of the prior one although it is always computable. Moreover, no spectral representation for the process is actually associated with this spectrum and this problem remains still open. Without such a representation, it would seem difficult to give some physical meaning to this spectrum.

\section{PROOFS}

Proof of proposition 1. For $t \leqslant 0$, we have $\beta_{t}^{X}(\cdot)=\beta^{(1)}(\cdot)$ and, for $t>0$,

$$
\beta_{t}^{X}(n)= \begin{cases}\beta^{(2)}(n) & \text { if } 0 \leqslant n<t \\ 0 & \text { if } n \geqslant t\end{cases}
$$


These equalities are obvious when $t \leqslant 0$ or $0 \leqslant n<t$. For $n \geqslant t>0$, it is easy to show that $\epsilon_{X}^{f}(t ; t-n+1)=\epsilon_{X^{(1)}}^{f}(t ; 1)$ and $\epsilon_{X}^{b}(t-n ; t-1)=\epsilon_{X^{(2)}}^{b}(t-n ; 0)$. These relationships leads to the result since these variables are uncorrelated.

ProOF of PROPERTY 7. The relationships

$$
\begin{aligned}
& \epsilon_{Y}^{f}(t ; t-n+1)=\mathrm{e}^{-\mathrm{i} \lambda_{0} t} \epsilon_{X}^{f}(t ; t-n+1) \\
& \epsilon_{Y}^{b}(t-n ; t-1)=\mathrm{e}^{-\mathrm{i} \lambda_{0}(t-n)} \epsilon_{X}^{b}(t-n ; t-1)
\end{aligned} \quad n \geqslant 1,
$$

show that $\beta_{t}^{Y}(n)=\mathrm{e}^{-\mathrm{i} \lambda_{0} n} \beta_{t}^{X}(n), n \geqslant 0$. We obtain recursively the relationships between the associated Szegö polynomials and also the equality of their norms:

$$
\left\{\begin{array}{l}
\varphi_{n}^{Y f}\left(\mathrm{e}^{\mathrm{i}\left(\lambda-\lambda_{0}\right)}\right)=\mathrm{e}^{-\mathrm{i} \lambda_{0} n} \varphi_{n}^{X f}\left(\mathrm{e}^{\mathrm{i} \lambda}\right) \\
\varphi_{n}^{Y b}\left(\mathrm{e}^{\mathrm{i}\left(\lambda-\lambda_{0}\right)}\right)=\varphi_{n}^{X b}\left(\mathrm{e}^{\mathrm{i} \lambda}\right) \\
\sigma_{Y}^{f}(n)=\sigma_{X}^{f}(n)
\end{array} \quad n \geqslant 0\right.
$$

Here to simplify the notation, the index $t$ is omitted. The norms equality leads to

$$
\begin{aligned}
\int_{-\pi}^{\pi} \varphi_{n}^{X f}\left(\mathrm{e}^{\mathrm{i} \lambda} \overline{\varphi_{m}^{X f}\left(\mathrm{e}^{\mathrm{i} \lambda}\right)} \mathrm{d} F_{t}^{X}(\lambda)\right. & =\int_{-\pi}^{\pi} \varphi_{n}^{Y f}\left(\mathrm{e}^{\mathrm{i} \lambda} \overline{\varphi_{m}^{Y f}\left(\mathrm{e}^{\mathrm{i} \lambda}\right)} \mathrm{d} F_{t}^{Y}(\lambda)\right. \\
& = \begin{cases}\sigma_{X}^{f 2}(n) & \text { if } n=m \\
0 & \text { otherwise }\end{cases}
\end{aligned}
$$

According to the relationships between the polynomials, we have

$$
\begin{aligned}
\int_{-\pi}^{\pi} \varphi_{n}^{X f}\left(\mathrm{e}^{\mathrm{i} \lambda}\right) \overline{\varphi_{m}^{X f}\left(\mathrm{e}^{\mathrm{i} \lambda}\right)} \mathrm{d} F_{t}^{X}(\lambda) & =\int_{-\pi}^{\pi} \mathrm{e}^{\mathrm{i} \lambda_{0}(n-m)} \varphi_{n}^{Y f}\left(\mathrm{e}^{\mathrm{i}\left(\lambda-\lambda_{0}\right)}\right) \overline{\varphi_{m}^{Y f}\left(\mathrm{e}^{\mathrm{i}\left(\lambda-\lambda_{0}\right)}\right)} \mathrm{d} F_{t}^{X}(\lambda) \\
& =\int_{-\pi-\lambda_{0}}^{\pi-\lambda_{0}} \varphi_{n}^{Y f}\left(\mathrm{e}^{\mathrm{i} \lambda} \overline{\varphi_{m}^{Y f}\left(\mathrm{e}^{\mathrm{i} \lambda}\right)} \mathrm{d} F_{t}^{X}\left(\lambda+\lambda_{0}\right)\right. \\
& =\int_{-\pi}^{\pi} \varphi_{n}^{Y f}\left(\mathrm{e}^{\mathrm{i} \lambda} \overline{\varphi_{m}^{Y f}\left(\mathrm{e}^{\mathrm{i} \lambda}\right)} \mathrm{d} F_{t}^{Y}(\lambda)\right.
\end{aligned}
$$

In the above expressions, the term $\mathrm{e}^{\mathrm{i} \lambda_{0}(n-m)}$ is deleted because the integral vanishes when $m$ is different from $n$. The result comes from the latter equality considered for all $(m, n)$.

ProOF OF PROPERTy 8 . When $Y(\cdot)=\overline{X(\cdot)}$, we have $\beta_{t}^{Y}(\cdot)=\overline{\beta_{t}^{X}(\cdot)}$. The relationships between the polynomials are obtained recursively and the norms are equal:

$$
\left\{\begin{array}{l}
\varphi_{n}^{Y f}(\bar{z})=\overline{\varphi_{n}^{X f}(z)} \\
\varphi_{n}^{Y b}(\bar{z})=\overline{\varphi_{n}^{X b}(z)} \\
\sigma_{Y}^{f}(n)=\sigma_{X}^{f}(n)
\end{array} \quad n \leqslant 0\right.
$$

Using the norms equality, the measure characterization leads to 


$$
\int_{-\pi}^{\pi} \varphi_{n}^{X f}\left(\mathrm{e}^{\mathrm{i} \lambda}\right) \overline{\varphi_{m}^{X f}\left(\mathrm{e}^{\mathrm{i} \lambda}\right)} \mathrm{d} F_{t}^{X}(-\lambda)=\int_{-\pi}^{\pi} \varphi_{n}^{Y f}\left(\mathrm{e}^{\mathrm{i} \lambda} \overline{\varphi_{m}^{Y f}\left(\mathrm{e}^{\mathrm{i} \lambda}\right)} \mathrm{d} F_{t}^{Y}(\lambda)\right.
$$

for all $(n, m)$. That proves the first assertion. As a consequence, the second holds. Notice that we can immediately obtain this latter result by pointing out that $\beta_{t}^{X}(\cdot)$ is real and hence $d F_{t}^{X}$ is symmetrical.

\section{ACKNOWLEDGEMENT}

The authors would like to thank a referee for his suggestions and for pointing useful references on the multivariate case.

\section{REFERENCES}

Burg, J. P. (1975) Maximum entropy spectral analysis. PhD dissertation, Dept. Geophys. Stanford Univ.

Cramér, H. (1961) On some classes of nonstationary stochastic processes. In Proceedings of 4th Berkeley Symposium on Mathematics, Statistic and Probability, vol 2, Berkeley: University of California Press, pp. 57-78.

Dégerine, S. (1990) Canonical partial autocorrelation function of a multivariate time series. Ann. Statist. 18, 961-71.

and Lambert-Lacroix, S. (1996) Evolutive instantaneous spectrum associated with partial autocorrelation function for nonstationary time series. Proceeding TFTS-96, 457-60.

and Lambert-Lacroix, S. (2002) Partial autocorrelation function of a nonstationary time series. J. of Multivariate Analysis, to appear.

Geronimus, Y. L. (1960) Polynomials Orthogonal on a Circle and Interval. New York: Pergamon.

Grenier, Y. (1984) Modélisation de signaux non stationnaires. Thèse de Doctorat d'État, Paris Sud.

Grenier, Y. (1987) Parametric time-frequency representations. In Signal Processing (eds J. L. Lacoume, T. S. Durrani and R. Stora) Les Houches: North-Holland, pp. 341-97.

Herbst, L. J. (1963) Periodogram analysis and variance fluctuations. J. Roy. Statist. Soc. Ser. B 25, 442-50.

Loynes, R. M. (1968) On the concept of the spectrum for non-stationary processes. J. Roy. Statist. Soc. Ser. B 30, 1-30.

Martin, W. and Flandrin, P. (1985) Wigner-Ville spectral analysis of nonstationary processes. IEEE Trans. Acoust., Speech Signal Process. 33, 1461-70.

Mélard, G. (1978) Propriétés du spectre évolutif d'un processus non stationnaire. Ann. Inst. H. Poincaré sec. B 14, 411-24.

and Herteleer-De Schutter, A. (1989) Contributions to evolutionary spectral theory. J. Time series Anal. 10, 41-63.

Priestley, M. B. (1965) Evolutionary spectra and non-stationary processes. J. Roy. Statist. Soc. Ser. B 27, 204-37.

and Tong, H. (1969) On the analysis of bivariate non- stationary processes. J. Roy. Statis. Soc. Ser. B 35, 153-66.

Ramsey, F. L. (1974) Characterization of the partial autocorrelation function. Ann. Statist. 2, 1296-301.

Tuøstheim, D. T. (1976) Spectral generating operators for non-stationary processes. Adv. Appl. Prob. 8, 831- 46.

Yule, G. U. (1907) On the theory of correlation for any number of variables, trated by a new system of notation. Royal Society Proceeding 79, 85-96. 